\title{
Tsafon
}

Revue d'études juives du Nord

73 | 2017

Connaissance de la Shoah : témoignages, enseignements, visites

\section{Présentation du dossier}

\section{Danielle Delmaire}

\section{(2) OpenEdition \\ Journals}

Édition électronique

URL : https://journals.openedition.org/tsafon/277

DOI : $10.4000 /$ tsafon. 277

ISSN : 2609-6420

Éditeur

Association Jean-Marie Delmaire

Édition imprimée

Date de publication : 1 juin 2017

Pagination : 7-28

ISSN : $1149-6630$

\section{Référence électronique}

Danielle Delmaire, «Présentation du dossier », Tsafon [En ligne], 73 | 2017, mis en ligne le 31 mai 2018, consulté le 30 juin 2021. URL : http://journals.openedition.org/tsafon/277 ; DOI : https://doi.org/ $10.4000 /$ tsafon. 277 


\section{Dossier}

rassemblé et présenté par Danielle Delmaire

\section{Connaissance de la Shoah}

Témoignages, enseignement, visites 
Tsafon 73 


\section{Préambule}

Trois volets constituent ce dossier qui étudie les moyens à mettre en œuvre pour connaître la Shoah, notamment parmi le public jeune fréquentant encore l'école :

- un volet "témoignages" avec deux témoignages recueillis auprès d'anciens déportés à l'âge de l'adolescence,

- un volet « enseignement » avec sept expériences d'enseignants dont six en France, à tous les niveaux de l'enseignement (école primaire, collège et lycée), et un en Allemagne,

- un volet "visites » de lieux de mémoire avec deux exposés sur les activités du Mémorial de la Shoah à Paris et de Kazerne Dossin à Malines en Belgique.

J'aurais aimé élargir le contenu des volets «enseignement» et " visites » de lieux de mémoire en associant, au moins, un enseignant israélien et l'institut Yad Vashem de Jérusalem. Hélas, après un premier contact qui me laissait espérer une telle collaboration, les relances et les rappels se sont avérés vains. Or il eut été fort intéressant de comparer ce qui se fait dans le «pays des coupables » et ce qui se fait dans le «pays des victimes »(A. Ballis). Néanmoins, avec les rapports d'expériences des six enseignants du nord de France, pays à la fois de coupables et de victimes, et l'excellent compte rendu d'A. Ballis pour l'Allemagne, quelques éléments de comparaison peuvent être élaborés.

Ce dossier n'a nullement la prétention, ni même une modeste intention, de tirer des conclusions définitives ou globalisantes. Six comptes rendus d'enseignants exerçant tous dans la même région de France et deux seuls exposés sur les activités de lieux de mémoire forment, bien évidemment, un corpus bien trop léger pour conclure définitivement. Il s'agit, ici, de quelques cas précis qui, sans être forcément particuliers, ne sont pas pour autant exemplaires. Entendre ces enseignants, écouter leurs élèves en me rendant dans leur classe, lire les 
exposés de travailleurs dans un lieu de mémoire sont autant d'approches qui permettent de comprendre comment les enfants et les adolescents prennent connaissance de la Shoah et comment ils réagissent à cette prise de connaissance. Dans cette démarche, qui ne se veut pas sociologique, $\mathrm{j}$ 'écoute et je constate. Je relève des points communs à ces rencontres et aussi des points de divergence mais le corpus est trop maigre pour m'aventurer au-delà de ces constats.

Pourquoi avoir tenté ces démarches ? En tant qu'universitaire ayant travaillé sur l'histoire des Juifs durant la Seconde Guerre mondiale dans le Nord de la France, sans pour autant avoir eu l'occasion d'enseigner ce sujet à mes étudiants dans leur cursus général en histoire, j'ai souvent été sollicitée, depuis une bonne vingtaine d'années, par des collègues de l'enseignement primaire mais surtout secondaire pour les aider à présenter la Shoah à leurs élèves. Soit, me demandait-on, pour donner des pistes d'exploitation documentaire, soit pour intervenir dans leur classe afin de parler de ce qui s'était passé dans le Nord, soit encore pour accompagner un rescapé de la Shoah qui était invité à témoigner devant les élèves. Ces derniers mois, je le fus particulièrement car plusieurs collègues préparaient une sortie vers un musée ou un voyage vers un lieu de mémoire et même vers Auschwitz. Prenant ainsi connaissance des efforts de ces collègues ${ }^{1}$ pour faire connaître la Shoah, l'idée m'est venue de confronter leurs expériences et de les rassembler dans ce dossier. Et je fus confortée, dans cette décision, par la lecture d'un article du n ${ }^{\circ} 56$, de janvier 2017, du Lien Francophone édité par Yad Vashem, intitulé «La Shoah et l'identité juive - défis de l'éducation » et qui rendait compte d'une conférence internationale organisée du 26 au 29 décembre 2016, à Yad Vashem par l'École Internationale pour l'Enseignement de la Shoah (EIES) « destinée aux directeurs d'écoles juives du monde entier » ${ }^{2}$. Des paroles d'Avner Shalev, président de Yad Vashem, y sont rapportées : «...notre défi majeur aujourd'hui est d'assurer une transmission de la mémoire munie d'un sens. Que les jeunes d'aujourd'hui puissent construire une identité en tant que Juif et en tant qu'Homme». Il ne s'agit pas, évidemment pour les élèves du nord de la France, de se « construire une identité en tant que Juif», mais de se construire « en tant qu'Homme» soucieux des droits de l'Homme et de la démocratie en

\footnotetext{
${ }^{1}$ Certains d'entre eux qui collaborent à ce dossier me sont devenus très proches et nous prolongeons nos rencontres en menant collectivement des recherches approfondies. Je les remercie ici pour cette complicité scientifique et amicale.

${ }^{2}$ Lire les pp. 2 et 3 de la revue.
} 
général et en tant que futur citoyen du monde, très certainement. Car, les échanges avec les élèves et leurs enseignants en témoignent, la connaissance de la Shoah instruit sur les événements de la Seconde Guerre mondiale mais surtout et aussi elle éduque et fait avancer sur le chemin d'un citoyen responsable de ses opinons et de ses engagements. En outre, «trois sujets » ont retenu l'attention des participants à cette conférence internationale : "l'enseignement de la Shoah en classe, les voyages de mémoire et la place du témoignage dans les programmes d'étude ». Les trois volets de ce dossier que j'étais en train de monter correspondaient exactement à ces «trois sujets». Seul l'ordre différait. En effet, il m'a paru plus pertinent de commencer par l'écoute de témoignages de rescapés avant d'aborder les expériences d'enseignants.

Je n'ouvre pas une porte. D'autres que moi ont écrit sur le sujet et précisément Didier Dumarque, philosophe, qui anima récemment un colloque «penser la Shoah » en partenariat avec le Mémorial de Caen et l'université populaire de la même ville, vient de publier Enseigner la Shoah, avec le sous titre "en 40 pages, ce que la Shoah enseigne » (édition uppr de Toulouse), ouvrage dans lequel le questionnement du sens de l'enseignement de la Shoah est clairement posé.

En 2010, dans son $n^{\circ}$ 193, la Revue d'Histoire de la Shoah a publié un épais volume (776 p.) sur le thème «Enseigner l'histoire de la Shoah» dans lequel l'évolution des programmes d'enseignement est analysée par divers auteurs, les pratiques et les moyens d'enseignement sont exposés, et parmi eux la littérature, les films, les visites de lieux comme le camp de Drancy ou la Maison d'Izieu où furent arrêtés une quarantaine d'enfants juifs pour être envoyés à la mort à Auschwitz, et enfin la situation de cet enseignement hors de France est présentée. L'ampleur de l'ouvrage incite à la réflexion.

D'autre part, Tsafon, dans son $\mathrm{n}^{\circ}$ 9-10 (été-automne 1992), consacré à «1942, l'année tragique des Juifs du Nord» a ouvert une rubrique intitulée : «Enseigner et connaître la Shoah» dans laquelle Frédéric Gugelot et Jean-Marie Delmaire faisaient part de leur expérience respective (pp. 100-110). Le dossier est mince et insiste sur la part émotionnelle qu'implique cet enseignement.

Bien sûr, la liste n'est pas exhaustive. Les conseils fournis dans les programmes pédagogiques (voir les contributions de M. Hebddebaut et R. Rigaut) attestent de l'importance de la bibliographie sur le sujet qui continue de retenir l'attention du ministère de l'Éducation nationale puisque le 27 janvier 2017, une cérémonie, placée sous le patronage de la 
ministre, s'est déroulée au Mémorial de la Shoah afin d'encourager des lycéens qui se font «ambassadeurs de la Mémoire ». Ces derniers ont reçu une formation, pour compléter leur connaissance de la Shoah, qui leur permet de mieux la diffuser ${ }^{3}$.

\section{Les témoignages de rescapés}

Le dossier restitue deux témoignages oraux, de Haïm Vidal Sephiha et de Charles Baron. Ils ont été prononcés lors d'un colloque qui eut lieu à l'université de Lille 3, en janvier 2005 et qui était consacré à l'ouverture des camps, soixante ans après ${ }^{4}$. Ces témoignages avaient été enregistrés mais n'avaient pas été publiés dans les actes du colloque, il m'a paru pertinent de les transcrire pour ce dossier. S'ajoutait le témoignage de Lili Leignel qui fut déportée à Ravensbrück alors qu'elle avait onze ans. Je n'ai pas rapporté son témoignage dans ce numéro de Tsafon car nous l'avions déjà publié dans une édition précédente ${ }^{5}$. Les deux témoins, tous deux rescapés d'Auschwitz et d'autres camps n'ont pas tout à fait vécu la même déportation. Haïm Vidal Sephiha ne fut pas libéré à l'ouverture du camp d'Auschwitz. Il insiste sur le fait que la libération des uns s'est accompagnée de la mort des autres, de ceux qui tombèrent lors des «marches de la mort», auxquelles il a survécu. Sa libération survient plusieurs longues semaines après. Charles Baron a connu Auschwitz mais il dit bien fort qu'il a eu " un sacré pot» car il a été transféré vers un autre camp avant l'arrivée des Soviétiques qui provoqua les départs précipités et les «marches de la mort». Il n'empêche qu'il aurait pu mourir plus d'une fois mais il a eu « du pot»!

Les trois témoins ont souvent parlé devant des élèves, notamment Charles Baron ${ }^{6}$ et Lili Leignel. Cette dernière reconnaissait, récemment,

\footnotetext{
${ }^{3}$ Compte rendu de la cérémonie dans Actualité Juive, ${ }^{\circ} 1423$ du 9 février 2017, p. 36.

${ }^{4}$ Les actes ont été publiés : Histoire et conscience. Il y a soixante ans, l'ouverture des camps d'extermination, (textes réunis par Nicole Abravanel, Martine BenoitRoubinowitz, Danielle Delmaire), Villeneuve d'Ascq, éditions du Conseil Scientifique de l'université Charles de Gaulle - Lille 3, 2007. Les détails biographiques des deux témoins sont à lire en notes dans leurs témoignages.

5 Tsafon, $\mathrm{n}^{\circ}$ 9-10, été-automne 1992, pp. 28-37. Je rappelle la déportation de Lili Leignel et de sa famille lors d'une intervention d'un élève de Catherine Bodet qui se souvient de l'avoir entendue en classe. Voir note 3 de la transcription de l'entretien avec les élèves de Catherine Bodet.

${ }^{6}$ Dans l'hommage que Tsafon a publié peu de temps après sa mort, n ${ }^{\circ} 72$ aut. 2016 hiv. 2017, nous avons reproduit, p. 156, une photo de Charles baron témoignant devant des élèves.
} 
avoir rencontré «plus de 13000 élèves chaque année, leur présence me réconforte $\rangle^{7}$. Pour ces témoignages incessants auprès de jeunes, Lili Leignel a obtenu une reconnaissance méritée en étant élevée au rang de chevalier de la Légion d'Honneur en 2011 et d'officier des Palmes académiques en 2016. Comme Charles Baron, Lili Leignel est toujours émue de constater la grande écoute des élèves lorsqu'elle évoque sa déportation à Ravensbrück puis Bergen-Belsen et son rapatriement en France avec ses petits frères. Tous deux savent créer une connivence avec leur auditoire. Il en est de même pour une autre rescapée de la déportation : Ida Grinspan qui serra une élève dans ses bras, lors d'une visite à Auschwitz (lire le rapport de voyage de L. élève de J-B. Gardon). Monique et Nicole Michel, petites filles juives réfugiées en Dordogne pour fuir la persécution dans le Nord, sont venues raconter leur histoire aux élèves de l'école de Flines-lez-Râches où elles avaient elles-mêmes étudiées. Elles ont également dialogué avec beaucoup de chaleur (rapport de M. Hedebaut). L'accueil est très souvent enthousiaste, les élèves confectionnent de grands panneaux souhaitant la bienvenue aux témoins (voir la photo de Charles Baron, $\mathrm{n}^{\circ} 72$ de Tsafon ou le panneau d'accueil réalisé par les élèves de la classe de $\mathrm{M}$. Heddebaut).

La qualité du témoignage dépend de l'écoute des élèves mais l'inverse est vrai aussi. L'attention s'intensifie non pas en déballant brutalement les atrocités mais en les présentant avec douceur, c'est souvent le cas de Lili Leignel que j'ai pu entendre plusieurs fois, voire avec humour pour détendre l'atmosphère, comme le font Haïm Vidal Sephiha et Charles Baron dans ce dossier. L'humour est utilisé aussi par contraste à l'horreur. Il peut être dérision quand Charles Baron remarque qu'il n'a pas de guide dans son train de déportation pour se repérer dans son « voyage »! L'humour peut encore être grinçant comme la répétition des mots "mamans» et "grands-mamans". Ils scandent le récit de Charles Baron qui décrit le triste cortège des déportés traversant les villages allemands, pour mieux pointer l'importance de l'éducation parentale qui entretient, chez les enfants, la haine de l'autre. Lili Leignel, pour adoucir son témoignage, termine souvent par une mélodie que les prisonnières de Ravensbrück chantaient pour se réconforter et par cette

\footnotetext{
${ }^{7}$ Propos rapportés par La Voix du Nord du 31 mars 2017, à propos du témoignage de Lili Leignel devant les élèves du Lycée professionnel René Cassin de Montigny-enOstrevent (Nord). D'autre part, Lili Leignel me confia que vingt ans après avoir entendu son témoignage, un enfant devenu adulte l'avait reconnue, lors d'une rencontre tout à fait fortuite, et il pouvait lui restituer son histoire sans hésitation. Cela l'avait beaucoup encouragée.
} 
mélodie, bien souvent, elle réconforte aussi son auditoire pris par l'émotion.

Les témoignages permettent une connaissance directe. L'histoire s'incarne grâce aux paroles des témoins, elle s'évade des livres et des cours du professeur. Même si Charles Baron s'appuie sur des documents d'archives pour illustrer et conforter son témoignage, sa parole donne un vécu auquel les élèves n'accèdent pas dans un cours. L'imprécision n'altère pas le témoignage (Haïm Vidal Sephiha ne se souvient plus du nom de la grange où les déportés firent une halte) en revanche un détail très précis habite le souvenir (Haïm Vidal Sephiha a retenu qu'il était le $161^{\mathrm{e}}$ déporté à monter dans le wagon, ses hallucinations et ses rêves frappent par la netteté du souvenir).

Les témoignages donnent une connaissance singulière de la Shoah mais non globale. Ce sont les cours des enseignants qui doivent mettre en place cette globalisation.

\section{L'enseignement de la Shoah}

Les sept enseignants qui ont accepté de participer à cette enquête ne professent pas dans un cadre identique même si six d'entre eux exercent dans la région lilloise du nord de la France.

M. Heddebaut est professeur des écoles dans un établissement primaire d'une petite ville, Flines-lez-Râches ; il porte bien le nom du grand résistant, juif, et défenseur des droits de l'Homme : René Cassin. La commune est une petite ville de 5500 habitants située entre deux villes plus importantes: Orchies et Douai. Les habitants y vivent minoritairement de l'agriculture mais plutôt de l'industrie environnante, des emplois de services et administratifs. La part de population immigrée $\mathrm{y}$ est très faible.

Les autres enseignants sollicités exercent dans des établissements secondaires : R. Rigaut en collège et J-B. Gardon ${ }^{8}$, C. Bodet, V. Beaumont et C. Duriez en lycée. Le collège Anne Frank, au nom également prédestiné pour une telle enquête, où enseigne R. Rigaut, se situe à Grande-Synthe (Nord) qui jouxte Dunkerque. La commune compte plus de 23000 habitants qui vivent en grande partie des activités industrielles et portuaires mais aussi des services. Elle doit sa notoriété à l'installation récente et controversée d'un camp de réfugiés.

\footnotetext{
${ }^{8}$ Il exerce actuellement dans un collège mais son rapport concerne un lycée.
} 
Les classes dans lesquelles je me suis rendue pour échanger avec les élèves ne se ressemblent pas. Le lycée de V. Beaumont, Marguerite de Flandre se situe à Gondecourt (Nord), commune rurale de 4000 habitants. Les élèves sont issus de ce milieu rural, de familles d'agriculteurs, de commerçants et/ou de fonctionnaires. Le lycée international Montebello où j'ai rencontré les élèves de C. Bodet et $\mathrm{C}$. Duriez a été récemment ouvert à Lille, en 1992, pour accueillir des élèves de familles modestes, parfois issues de l'immigration, résidant dans les quartiers populaires de Lille-sud et de Wazemmes. En même temps, sa création répondait aussi à une politique de mixité sociale. Des classes sont intégrées dans un partenariat avec des établissements étrangers et neuf langues étrangères sont enseignées. Le lycée devint international en 2014. C'est un grand établissement fréquenté par 1400 élèves, selon son site, se répartissant dans des classes d'enseignement général (L, ES, S) et technique (STMG ou sciences et technologies du management et de la gestion). La classe de $\mathrm{C}$. Bodet, une Première $\mathrm{S}$, a la réputation d'être « une bonne classe » c'est moins le cas de la classe de C. Duriez qui m'avait prévenu que ses élèves n'étaient pas du même niveau que ceux de la classe de sa collègue. En effet, cette Première STMG eut un comportement plus agité mais l'écoute n'en fut pas moindre, en revanche la discussion fut plus dispersée. C'est uniquement dans cette classe, où la proportion d'élèves issus de l'immigration était plus importante, que l'échange dériva vers la comparaison entre Shoah et esclavage des Noirs.

Les élèves de cette classe avaient à peu près le même profil que ceux de la classe de J-B. Gardon à Roubaix. Le lycée Jean-Moulin de Roubaix dispense un enseignement général, technologique et professionnel. Ce sont donc aussi des élèves moins brillants, mais non moins intéressants, qui avaient accepté le projet de leur professeur de se rendre à Auschwitz. Je ne me suis pas rendue dans cette classe ; J-B. Gardon a livré un rapport sur son expérience de l'enseignement de la Shoah complété par un voyage au camp d'extermination des Juifs. Le projet ne fut pas d'emblée bien accueilli par ces élèves dont une bonne partie était aussi issue de l'immigration avec une proportion non négligeable de musulmans car la ville de Roubaix est connue pour avoir un grand nombre de musulmans parmi ses 9500 habitants. La ville a beaucoup souffert de la fermeture des établissements textiles et de la réduction d'activité de grands centres de vente par correspondance. La ville a un long passé ouvrier et socialiste voire collectiviste (Jules Guesde en fut député à la fin du $\mathrm{XIX}^{\mathrm{e}}$ siècle). Toutefois en ce début du $\mathrm{XXI}^{\mathrm{e}}$ 
siècle, la ville se heurte aux problèmes d'insertion professionnelle d'une part de ses habitants. Dans la classe de J-B. Gardon, ces difficultés apparaissaient.

Enfin, quant à A. Ballis, elle n'enseigne pas dans les établissements secondaires de l'Allemagne mais en tant que titulaire de la chaire de didactique de langue et littérature allemandes et de l'allemand comme langue seconde à l'université Louis-et-Maximilien de Munich, son expérience peut être utile. Elle livre des informations qui méritent une comparaison avec ce qui est enseignée en France et surtout en Israël. J'y reviendrai dans la dernière partie de cette présentation. Surtout, elle renseigne sur la politique d'éducation à la Shoah et sur les programmes, en RFA puis en Allemagne, ainsi que sur la réaction d'enseignants et d'élèves.

Mais l'enseignement n'est pas la seule voie pour connaître la Shoah. L'Histoire doit sortir de la salle de classe. Il reste donc les visites des lieux de mémoire qui entretiennent le souvenir de cet enseignement et le renforcent.

\section{Les visites des lieux de mémoire}

Les deux instituts sollicités sont des lieux de mémoire qui renferment chacun un musée évoquant la Shoah en France, pour le Mémorial de la Shoah à Paris, et en Belgique, pour Kazerne Dossin à Malines.

Le premier n'est pas un lieu où les Juifs furent internés mais très souvent la visite du musée du Mémorial est couplée avec celle du musée de Drancy qui fait face à la Cité de la Muette où furent rassemblés des milliers de Juifs arrêtés en France, avant leur départ pour Auschwitz. Le Mémorial de Paris, tel qu'il existe actuellement, fut inauguré en janvier 2005. Il agrandit le vieux bâtiment inauguré en 1957 et qui abritait le Mémorial du martyr juif inconnu ainsi que le Centre de Documentation juive contemporaine (CDJC créé clandestinement en 1943) qui contient toutes les archives concernant la déportation des Juifs en France. En outre, l'ensemble présente le mur des Noms (de tous les déportés juifs partis de France), le mémorial des enfants et, à l'extérieur, le mur des Justes parmi les Nations de France.

Le second est un musée sur la déportation des Juifs mais qui s'ouvre aussi sur l'histoire des Droits de l'Homme, il fait face à la caserne elle-même où furent enfermés les déportés juifs et tsiganes. Le 
bâtiment du musée est récent, il fut ouvert au public en décembre 2012. Auparavant, musée et archives étaient hébergés étroitement dans une seule aile de la caserne dont les trois autres parties furent aménagées, en 1980, en logements assez luxueux.

Les deux établissements accueillent des visiteurs et, parmi eux, majoritairement des groupes scolaires. Au Mémorial de la Shoah de Paris, les professeurs de tous niveaux peuvent même suivre, en été, une formation pédagogique pour leur enseignement. Ils sont aussi invités, avec leurs élèves, à une visite préparatoire aux voyages vers les camps d'Europe de l'est. Comme pour Kazerne Dossin, le but du Mémorial est de former, instruire pour mieux transmettre l'histoire de la Shoah. Le guide de Kazerne Dossin (D. Laboux), le responsable des voyages du Mémorial de Paris (M. Orjekh) et les enseignants qui ont profité des visites (B. Bossy, J-B. Gardon, R. Rigaut) s'accordent pour reconnaître que les visites peuvent compléter les lacunes de l'enseignement dispensé en classe. En outre, ils sont aussi le lieu où il est possible, pour les élèves, de rencontrer un rescapé de la déportation et de dialoguer avec lui. Là encore, sur ces lieux, l'Histoire s'incarne. Elle sort des livres d'histoire scolaires.

\section{Les points communs entre les enseignants et les guides des musées}

De cette consultation pour déterminer les moyens de connaître l'histoire de la Shoah, apparaissent quelques points communs aux intervenants (enseignants et guides des musées) ainsi que dans la prise de paroles par les élèves.

Autres moyens de connaissance que l'enseignement

Bien d'autres moyens que le cours en classe ont été utilisés par les enseignants. Quant aux élèves, quelques uns disent avoir pris une première connaissance de la Shoah ailleurs qu'en classe.

Ils ont vu des films (surtout dans la classe de Première STMG de C. Duriez) qu'ils soient des documentaires, des restitutions de parcours de déportés (La Liste de Schindler) ou des fictions (La vie est belle, film qui a souvent été cité alors qu'avec des professeurs je confiais ma réticence à l'égard de ce film mais les élèves ont intelligemment rétorqué que la fiction peut s'éloigner de l'histoire et peut être utile pour attirer l'attention sur le sujet). 
Les livres sont également un moyen de connaissance, il peut s'agir de documentaires ou de témoignages. Le journal d'Anne Frank a bien sûr souvent été cité. Les livres ont pu être étudiés en classe ou lus par les élèves sans obligation scolaire. Un élève de la classe de V. Beaumont a même fait part de l'influence familiale dans son choix de livres ou de films. Et dans son inventaire des moyens pour connaître la Shoah, l'élève de $\mathrm{V}$. Beaumont qui répond au questionnaire après le voyage à Auschwitz cite : les films, les livres, les témoignages oraux, les visites de musées ou de mémoriaux et, ajoute-t-elle, les commémorations auxquelles personne d'autre (enseignants ou élèves) n'a fait référence.

Enfin A. Ballis, pour l'Allemagne, fournit des renseignements précieux et précis sur l'utilisation de vidéos en y ajoutant des conseils pour mener à bien une pédagogie de la Shoah. Elle incite également à l'utilisation de site web'.

Importance d'une histoire incarnée

Plusieurs enseignants et les élèves ont insisté sur le rôle de la présence de témoins pour compléter leurs cours (R. Rigaut, $M$. Heddebaut, J-B. Gardon, V. Beaumont) ou encore sur l'importance de la lecture du Journal d'Anne Frank.

C'est alors que le récit s'incarne dans une personne que l'on rencontre ou dont on lit le journal. La conversation avec un témoin jette tous les doutes à terre. L'élève de la classe de J-B. Gardon, qui tenait tête à son enseignant et entraînait ses condisciples dans la contestation, se laisse fléchir lorsqu'elle entend le témoignage de Charles Baron : "Si le témoignage de Charles Baron a fait basculer la classe, la biographie de Pierre Vidal-Naquet (décédé en juillet 2006) a porté l'estocade » rapporte J-B. Gardon. Et l'entrevue avec Ida Grinspan sur le lieu même de la détention la bouleverse et en fait une « ambassadrice» de l'histoire de la Shoah. «L'histoire s'incarnait » écrit J-B. Gardon ou bien, rapporte R. Rigaud «...les victimes de cet assassinat de masse sont des êtres incarnés, avec un nom, un visage, une histoire ». Et un élève de V. Beaumont reconnaît : "On sent l'émotion de la personne quand elle

\footnotetext{
${ }^{9}$ À ce sujet, il importe de prendre connaissance de l'existence d'un nouveau site internet en français de Yad Vashem, inauguré le 23 mars 2017, sur lequel le visiteur peut découvrir des témoignages, des films d'archives, l'historique des convois partis de France, des renseignements sur les Justes de France, des expositions virtuelles etc. C'est une source multiforme pour la connaissance de la Shoah. Voir le site: www.yadvashem.org/fr. Cf. Yad Vashem, Le Lien Francophone, n 57, avril 2017.
} 
raconte puisqu'elle l'a vécu. Ça nous touche. Le fait de l'avoir vécu, c'est différent dans la façon de raconter ». Le récit du témoin atteint l'auditoire plus profondément que celui de l'enseignant

A. Ballis met aussi l'accent sur l'importance de l'histoire orale car le témoin «apporte la preuve que tout ce qui a été enseigné à l'école a réellement eu lieu».

\section{L'histoire locale sous tend l'histoire générale}

Dans les classes où j'ai pu présenter l'histoire du sort des Juifs de Lille durant la guerre avec projection de documents d'archives, l'intérêt pour l'histoire locale a été manifeste (C. Bodet, V. Beaumont, C. Duriez). Comme l'histoire touche parce qu'elle s'incarne par le témoignage d'un rescapé, elle touche encore car elle devient proche ou voisine lorsqu'on évoque les lieux, les rues, les maisons, les gares où des Juifs de Lille ont été arrêtés, ont été embarqués ou ont vécu cachés. L'histoire s'est passée près des élèves. À Gondecourt (V. Beaumont) où l'ancien collège avait caché deux petites filles juives, cette histoire locale a atteint une grande proximité.

Il en est de même pour les élèves de M. Heddebaut qui, avec leur professeur, découvrent que la famille juive Michel habitait leur ville, dans leur rue, et que leur entreprise fut « aryanisée ». Quant à B. Bossy, il rend compte de l'éveil à l'histoire locale après un voyage à Auschwitz. La Shoah ne s'est pas déroulée uniquement dans les lointaines contrées d'Europe de l'est mais elle fait partie de l'histoire locale " proche de [l']univers géographique » des élèves ( $M$. Orjekh). Et un élève de C. Bodet affirme que maîtriser l'histoire locale : «C'est plus impactant car on connaît les lieux où ces arrestations se sont passées, on est passé à ces endroits. On a une autre vision de la Shoah ». Et un autre d'ajouter, toujours à propos de l'exposé sur ce qui s'est passé à Lille : «Oui avec cet exposé, on a vu autre chose qu'Auschwitz et c'est bien ».

De l'intérêt des voyages à Auschwitz, ou d'autres camps ?

Selon A. Ballis, les visites de camps sont fréquentes dans les établissements secondaires en Allemagne. Elles sont souvent subventionnées par les Länder tant leur intérêt pédagogique est évident : «Comme sous l'effet d'une loupe, les objectifs pédagogiques semblent se concentrer en un faisceau lors d'une telle visite» commente 
l'universitaire de Munich. Dans certains camps, en Allemagne, des « objets archéologiques » sont exposés pour encore mieux appréhender le quotidien des prisonniers, c'est le cas à Buchenwald.

M. Orjekh, responsable de l'organisation des voyages au sein du Mémorial de la Shoah à Paris, insiste sur l'importance de la préparation au voyage. Avec leur enseignant, les élèves sont invités à venir s'informer au Mémorial avant le voyage. C'est ce qu'ont fait B. Bossy et J-G. Gardon qui ont réalisé ces sorties. La préparation du voyage est indispensable pour la réussite pédagogique de la visite. V. Beaumont avait préparé ses élèves au choc éprouvant de la vision du camp en m'invitant à leur parler de la Shoah et du site d'Auschwitz. Selon le mot de l'historienne Annette Wieviorka, citée par R. Rigaut, il faut savoir « ce qu'il y a à voir » à Auschwitz et c'est durant le cours d'histoire que doit aussi se faire la préparation à la visite d'un camp se convainc R. Rigaut. J-B. Gardon se range à cet avis car selon lui : «Être présent à Birkenau n'apporte rien [aux] élèves. C'est le cheminement et le questionnement qui nous a semblé important ». Une fois bien préparée, la visite d'un camp favorise la compréhension des faits par les élèves, comme l'écoute d'un témoin capte leur attention. «Ce qu'on raconte pendant la visite [du camp du Struthof en Alsace], quand on voit le four crématoire, c'est là qu'on se rend compte, vraiment, de la violence des choses » explique un élève de V. Beaumont.

La préparation, en amont de la visite, est donc primordiale mais la réflexion ou la restitution du voyage, en aval, s'avère tout autant nécessaire. Les élèves ne doivent pas rester sans soutien après avoir subi la vision de l'horreur. C'est la raison pour laquelle le Mémorial de la Shoah reste en contact avec les enseignants, au retour du voyage, et leur demande de rédiger des comptes rendus ou d'entreprendre des actions pour entretenir la mémoire. Ce que firent B. Bossy, V. Beaumont et R. Rigaut : leurs élèves ont travaillé le sujet de la Shoah et en ont fait une présentation à leurs condisciples ou à un public élargi.

Pour autant, selon R. Rigaut : «...l'intervention d'un témoin ou l'organisation de tels voyages ne présentent aucun caractère obligatoire... En d'autres termes, il n'est pas impératif d'aller à Auschwitz pour comprendre la Shoah !». Surtout lorsque le voyage est mal préparé. C'est ce que déplore $\mathrm{C}$. Bodet : «J'ai emmené des élèves à Auschwitz, il y a une dizaine d'années... Le voyage s'est effectué sur une journée et a commencé à 3 heures du matin. Nous sommes arrivés crevés, puis il a fallu prendre un bus de Cracovie à Auschwitz... Les 
élèves étaient fatigués, énervés, certains ont ri et ils ont été grondés. [La visite] a été éprouvante. C'est pour cela qu'une visite d'Auschwitz, c'est bien mais ce n'est pas bien ». L'enjeu exige donc une excellente préparation et des conditions de visite tout aussi excellentes.

Et finalement, conclut R. Rigaud, la visite d'un lieu ou un voyage ne peuvent pas remplacer un cours. Les deux se complètent.

\section{L'émotion suscitée par le sujet}

Tous, enseignants, guides, élèves en conviennent le sujet suscite une forte émotion. C'était aussi le constat de Frédéric Gugelot dans le récit de son expérience rapportée dans le $\mathrm{n}^{\circ}$ 9-10 de Tsafon (voir plus haut). À l'écoute d'un récit, à la vision d'un film, des gorges peuvent se nouer, les larmes peuvent perler. "C'est choquant» se sont exclamés plusieurs élèves de C. Duriez et ceci à plusieurs reprises. « Sa dimension tragique [de la Shoah], l'énormité des chiffres dépassent l'entendement. Tout concourt à lui donner une charge émotionnelle qui pourrait submerger les élèves, mais également les enseignants » écrit $M$. Heddebaut. Et R. Rigaut pose la question : « ...comment gérer l'émotion suscitée par ce témoignage bouleversant, délivré sur les lieux du drame ? » à propos du témoignage fait par Jacques Saurel au camp même de Bergen Belsen où il fut détenu. Pour les enseignants comme pour les élèves : «Être capable de dépasser cette émotion légitime pour penser l'événement est un exercice intellectuel difficile mais pourtant indispensable » (R . Rigaut).

Faut-il donc réserver la connaissance de la Shoah à des élèves plus avancés en âge ? Une partie des élèves que j'ai rencontrés pensaient qu'il ne fallait pas enseigner la Shoah à des élèves des classes primaires. Ce que pourtant a réussi à faire $\mathrm{M}$. Heddebaut, leur ai-je répondu. Comme elle, je suis convaincue que l'on peut faire connaître la Shoah à des enfants d'une dizaine d'années à condition de veiller à ne pas les choquer car comme le précise M. Heddebaut: «Dans l'enseignement de l'histoire, même à l'école élémentaire, il [l'enseignement de la Shoah] est devenu un moment fort, jamais anodin, mais qui ne peut être abordé comme n'importe quel autre événement particulier de l'histoire contemporaine ». Une élève de $\mathrm{V}$. Beaumont a clairement exposé la nécessité de nuancer les propos en fonction de l'âge des élèves : « $\mathrm{La}$ première fois, on nous en parle très vaguement dans l'enseignement primaire, puis au collège on nous raconte plus précisément et on a plus 
conscience, plus de connaissance des faits... Alors on réalise vraiment. Je trouve que la prise de conscience du côté humain des événements intervient plus en vieillissant ».

Quoi qu'il en soit et quel que soit l'âge des élèves, à l'écoute des témoins, à la lecture des récits, l'émotion est toujours présente. Il faut donc se garder d'en rester aux sentiments pour connaître et comprendre l'histoire de la Shoah. Tel est l'avis de M. Heddebaut: "Mais si l'enseignement de la Shoah fait appel à l'affectif, il ne peut à lui seul donner la matière d'un cours d'histoire, il ne peut le remplacer. Se contenter de l'émotionnel n'est pas faire de l'histoire » rappelle-t-elle.

\section{L'indispensable interdisciplinarité}

Pour M. Heddebaut, professeur des écoles, il est aisé de solliciter plusieurs disciplines pour parler de la Shoah, car elle les pratique toutes dans une seule classe et ceci durant une journée entière, ne se trouvant pas limitée à un cours d'une heure. La situation est différente pour un professeur des collèges ou des lycées qui partage ses classes avec d'autres collègues et n'enseigne qu'une discipline pendant une heure.

R. Rigaut et J-B. Gardon ont partagé leur projet avec des collègues de français prioritairement mais R. Rigaut a obtenu l'aide d'enseignants d'autres disciplines (allemand, musique, art, etc.). J-B. Gardon s'est même senti très soutenu par sa collègue de lettres qui a adhéré à sa démarche : «Sans ma collègue je n'aurai jamais tenté cette expérience, et elle, sans moi, non plus je pense. Au-delà de l'interdisciplinarité, j'ai la conviction qu'il faut être a minima deux pour se lancer dans ce type de projet, avoir une vraie relation de confiance et d'estime réciproque ». Les trois exemples de projet présentés par B. Bossy ont tous été menés par plusieurs enseignants de disciplines différentes.

A. Ballis, pour l'Allemagne, préconise également: «...une approche interdisciplinaire ainsi qu'une coopération étroite avec des institutions extrascolaires ». Le projet n'en est que plus attractif.

\section{Les points particuliers}

Prise de conscience de la négation de la Shoah

Dans une seule classe, le thème négationniste a été abordé mais pas spontanément : ce fut l'enseignante, C. Bodet, qui a lancé le débat sur le 
sujet. Unanimement, les élèves ont reconnu l'existence des négateurs et s'en sont montrés hostiles voire indignés. Le sujet n'ayant pas été abordé ailleurs, cela ne signifie pas que les élèves des autres classes n'y sont pas sensibles. À cette occasion, C. Bodet eut la présence d'esprit de remarquer que l'on ne «croit» pas en la Shoah comme l'on peut adhérer à une croyance spirituelle: «...peut-on ne pas croire à une vérité historique, et qui a été largement prouvée ?» interrogea-t-elle. Question qui interpella ses élèves.

La classe peu réceptive au sujet

Sur les six enseignants de la région lilloise sollicités pour ce dossier, deux ont dû batailler pour persister dans leur volonté de faire connaître la Shoah.

Dans la classe de C. Duriez, nous n'avons pas vraiment rencontré d'hostilité mais un reproche de taille : l'enseignement de la Shoah prend trop d'importance et occulte d'autres sujets d'histoire tout aussi dramatiques comme l'esclavage des Noirs ou la déportation des Tsiganes. La conversation n'a pas d'emblée glissé vers ce reproche, ce n'est qu'au bout d'une bonne demi heure qu'un élève a estimé qu'un cours sur le génocide des Juifs ne devrait pas occuper une place particulière. Il n'avait pas pris la parole auparavant et il m'a semblé qu'il avait rongé son frein pendant la demi heure précédente avant d'entrer dans le débat qui animait ses camarades. Il prolongea son propos assez loin puisqu'il mit en doute certains aspects de l'histoire même de la Shoah: «Vous accentuez sur les Juifs par rapport aux autres cours mais il y en a qui disent beaucoup de bêtises » lança-t-il. Il faisait allusion à ma critique envers La vie est belle, film de fiction dans lequel des invraisemblances m'avaient dérangée. J'ai alors pris conscience du danger des inventions de la fiction à propos d'un tel sujet car pourquoi admettre la réalité des faits si ceux-ci sont inventés ? La fiction peut-elle nourrir la négation?

Le reproche de l'élève fut accepté par une grande partie de sa classe et environ une dizaine d'élèves, majoritairement Noirs, mirent en concurrence les mémoires, celle de l'esclavage des Noirs et celle du génocide des Juifs. Il m'a paru alors impératif de préciser le sens des mots. En effet, la déportation des Noirs vers le Nouveau Monde et leur esclavage sont-ils identiques à la déportation des Juifs vers les camps d'Europe de l'est et à leur extermination ? La discussion commença à 
s'orienter en ce sens mais, manifestement, le manque d'informations précises sur ces deux déportations malmenait l'échange. Il aurait fallu plusieurs exposés sur chacune d'elles pour mener une conversation plus sereine. Le temps manqua, l'enseignant et moi ne disposions que d'une heure.

On mesure alors les efforts épuisants déployés par J-B. Gardon, et sa collègue, pour aborder l'enseignement, obligatoire, de la Shoah dans une classe en situation difficile, d'un lycée de Roubaix. Le contenu même de son cours fut remis en cause par une partie de ses élèves menés par une jeune fille aux propos franchement antisémites et niant la légitimité de l'État d'Israël. L'enseignant ne renonça pas et persista dans son projet de préparer ses élèves à un voyage à Auschwitz, en partenariat avec sa collègue de lettres. Ses rappels constants aux valeurs républicaines, contraires à toute forme de racisme, et au droit républicain qui condamne pénalement toute expression raciste, rappels étayés par des témoignages, finirent par avoir raison de l'occultation de l'histoire de la Shoah revendiquée par ces élèves.

Mais si la bataille fut gagnée par l'enseignant, elle fut bien rude : «Ma collègue et moi-même sortions parfois de cours épuisés par une heure de "combat»: tenir bon, rappeler la règle, répondre aux provocations et déconstruire les stéréotypes inlassablement » rapporte JB. Gardon.

Le cas particulier de l'Allemagne

L'enseignement de la Shoah auprès de jeunes Allemands pose inéluctablement un problème d'identité explique A. Ballis. L'Allemagne fut « le pays des coupables» et les Allemands furent les bourreaux. Actuellement, être un Allemand signifie-t-il être coupable ? Et, dans ce passé tragique, faut-il mettre en cause les grands-parents ? «...le travail de mémoire [a] profondément marqué l'image que l'État allemand a de lui-même ainsi que son identité nationale » affirme A. Ballis. Il s'agit donc d'enseigner ce passé et de l'assumer pour mieux se projeter dans l'avenir. La volonté vient d'en haut car c'est bien dans ce sens que sont prononcés les discours des présidents de la République fédérale.

Les programmes officiels d'enseignement imposent donc la connaissance de la Shoah mais donnent à réfléchir sur l'image de soi que renvoie cette connaissance. La connaissance des crimes doit faire émerger une reconnaissance de la responsabilité allemande mais en 
prenant garde à ne pas susciter des sentiments irraisonnés de refus ou de trop grande culpabilité ; dans les deux cas la faute serait trop lourde à porter. En Allemagne, plus qu'ailleurs, l'éducation doit donc éveiller l'esprit critique chez les élèves afin d'avoir la capacité d'adopter une attitude démocratique et responsable.

Ces dernières années, l'unification a dû compliquer la tâche des enseignants, me semble-t-il, d'une part à cause du voisinage accepté de l'Allemagne de l'est dont les habitants étaient habitués à rejeter la responsabilité des crimes sur l'autre Allemagne; d'autre part à cause du plus grand nombre d'élèves issus de l'immigration pour lesquels le sentiment de culpabilité n'a aucun sens. Ce passé n'est pas le leur.

On peut encore ajouter, à la réflexion d'A. Ballis, que si la connaissance de la Shoah peut engendrer des conflits de génération dans les familles allemandes, il peut en être de même dans les familles de pays occupés par les nazis où des collaborateurs ont aussi été coupables de crimes. Ce fut le cas, par exemple, de la famille du philosophe et essayiste Pascal Bruckner qui milita, dans les courants de la gauche française, contre l'antisémitisme en extraordinaire opposition à son père, antisémite et pronazi dès la fin des années trente et jusqu'à son décès en $2012^{10}$.

Prolongeant la démarche d'A. Ballis, il faut admettre que la problématique donne aussi à réfléchir en Israël où vit une grande partie des victimes et devient ainsi «le pays des victimes»! La conférence internationale organisée par l'EIES, dont j'ai parlé dans le préambule, place ses intervenants devant la question de l'identité : un Juif doit-il nécessairement s'identifier à une victime de la Shoah ? Dans le compte rendu de cette conférence ${ }^{11}$, on peut lire : «Le message principal de cette conférence était de faire prévaloir dans l'enseignement juif d'aujourd'hui une identité juive positive construite sur la richesse de la culture, de l'éthique, de la religion, de la tradition juive et non sur Auschwitz » (p. 2). Et le chroniqueur résume ainsi les paroles de Shulamit Imber, directrice pédagogique de l'EIES: «...la Shoah porte une place considérable dans l'histoire juive, une page que nous ne pouvons ignorer ou éviter... La Shoah ne doit pas non plus être un raccourci pour une

\footnotetext{
${ }^{10}$ Lire son autobiographie, Un bon fils, Paris, Grasset, 2014. Son engagement ferme contre l'antisémitisme lui valut même un procès en diffamation de la part d'associations qui veillent à enrayer toutes formes d'« islamophobie », le 30 novembre 2016. Les dites associations ont été déboutées par le verdict du 17 janvier 2017.

${ }^{11}$ Paru dans le n 56 de janvier 2017 de Yad Vashem, Le Lien Francophone, pp. 2-3.
} 
identité plus juive ou universelle ». Dans tous les établissements scolaires israéliens est diffusé un enseignement de la Shoah. De nombreux établissements et institutions en conservent la mémoire : Yad Vashem ou Lohamei Hagettaot pour ne citer que les deux plus célèbres. Par ailleurs, chaque université possède un centre de recherches sur la Shoah de sorte que chaque étudiant en histoire a pu intégrer, dans son cursus, une connaissance de la Shoah qu'à son tour il devra transmettre.

L'on comprend aisément que les pédagogues et historiens d'Allemagne et d'Israël coopèrent dans ce contexte particulier d'identification individuelle en fonction du passé familial et qu'A. Ballis attache de l'importance à la collaboration avec Yad Vashem. En fait, cette problématique d'indentification devrait aussi se poser accessoirement dans les autres pays où des collaborateurs ou de simples délateurs ont également commis des crimes. En France, tout le monde n'a pas résisté et des Juifs ont été dénoncés.

\section{Conclusion}

Ce dossier à trois volets n'a nullement la prétention d'avoir fait le tour de la question concernant la connaissance de la Shoah, ni non plus de donner des conclusions définitives. Modestement, mon souhait se limite à présenter des témoignages inédits et quelques expériences d'enseignement et de visites de lieux de mémoire. L'on pourrait ajouter d'autres canaux permettant de connaître la Shoah, comme l'utilisation des arts : la peinture, la sculpture, la musique, les chants... L'on pourrait encore évoquer des initiatives prises dans le cadre d'associations s'adressant à des élèves. Par exemple, il me plaît de présenter le travail accompli par la municipalité Les Mazures (Ardennes, France) où se trouvait un Judenlager durant la Seconde Guerre mondiale ${ }^{12}$. En partenariat avec l'association du Judenlager des Mazures, la mairie a réussi à mettre en place un "Chemin de la Mémoire ${ }^{13}$ et envisage des

\footnotetext{
${ }^{12}$ Dans ce Judenlager furent internés 288 Juifs de Belgique, astreints à un travail de forçat, durant tout l'été 1942, pour fabriquer du charbon de bois pour le compte de l'Organisation Todt. Une première déportation vers Auschwitz, par Malines, vide une partie du camp. D’autres prisonniers furent déportés par Drancy le 4 janvier 1944. Sur 288 prisonniers, 237 ne sont pas revenus de déportation, 2 furent fusillés pour évasion, 22 réussirent leur évasion, aidés par la Résistance locale, et 27 ont survécu. Lire JeanÉmile Andreux, «Mémorial des déportés du Judenlager des Mazures », Tsafon hors série $\mathrm{n}^{\circ} 3$, octobre 2007 .

${ }^{13}$ Lire le compte rendu de l'inauguration du «Chemin de la Mémoire » dans Tsafon, $\mathrm{n}^{\circ} 72$, automne 2016-hiver 2017, pp. 170-171.
} 
actions pédagogiques à partir de ce lieu de mémoire. Ainsi, une enseignante de l'école primaire du village encadre ses élèves pour faire aboutir un échange épistolaire avec un rescapé du camp qui vit maintenant au Canada. À la fin de cette année scolaire, un cahier confectionné par les élèves devra restituer l'historique de cette action, augmenté du récit des souvenirs d'un évadé du camp, grâce à sa fille qui collabore au projet pédagogique.

Décidément ce dossier est bien incomplet, il faut le reconnaître et, finalement, le sujet pourrait sans doute faire l'objet d'une réflexion s'étalant sur plusieurs jours d'un colloque.

Toutefois, par ce dossier, nous pouvons constater que les jeunes consultés, ainsi que leurs enseignants, sont quasiment unanimes pour admettre que la connaissance de la Shoah les aide à devenir des adultes critiques à l'égard des idéologies et responsables de leurs actes. «...on réalise vraiment ce qui s'est passé et ça nous permet aussi de nous forger nos opinions politiques, c'est vraiment à partir de ce moment-là qu'on peut, comment dire... qu'on réalise vraiment et qu'on arrive à avoir une opinion sur le monde actuel ! » reconnaît un élève de V. Beaumont.

Les élèves ont également pris conscience que désormais ils ont une connaissance, un savoir à transmettre. Ils deviennent des passeurs de mémoire ou des « ambassadeurs » de cette mémoire comme s'exprime le réseau des lieux de mémoire du Mémorial de la Shoah. L'élève de V. Beaumont, qui participa au voyage à Auschwitz, parle même de mission à accomplir : «En effet, après avoir eu la chance de me rendre sur les lieux du génocide juif et tzigane, je me dois de continuer à transmettre la mémoire des événements passés. C'est aussi la mission que nous a confiée $\mathrm{M}^{\text {me }}$ Lili Leignel...». Cette pratique d'un voyage à Auschwitz pour perpétuer le souvenir de la Shoah est très utilisée par les responsables pédagogiques israéliens nous informe A. Ballis : "Chaque année, 30000 jeunes Israéliens, accompagnés de leurs professeurs d'histoire, se rendent en Pologne pour transmettre aux générations futures le souvenir de l'Holocauste ».

Le plus difficile reste à persuader les élèves récalcitrants à cet enseignement, souvent parce qu'ils sont originaires (eux ou sinon leur famille) de pays qui n'étaient pas impliqués dans la guerre et donc dans l'extermination des Juifs et des tsiganes. Cette histoire : « ...c'est aussi jusqu'à un certain point leur histoire », tente de les convaincre J-B. Gardon, car ajoute-t-il «...à Tréblinka, Maïdanek ou Birkenau, c’est l'humanité que l'on a assassinée » et encore «...parce que l'école 
républicaine a été présente pour ces enfants juifs, hier, comme elle est présente, aujourd'hui, pour eux». Et pour cet enseignant dans un établissement difficile, il n'y a pas de «Territoires perdus pour la République. Il y a parfois des territoires qui manquent de républicains. À nous, enseignants, de les former $»^{14}$. Autrement dit, si des territoires peuvent paraître «perdus », ils sont récupérables. Mais au prix de quels efforts et de quels affrontements épuisants qui peuvent décourager plus d'un!

Ainsi, cent ans après les « hussards noirs » de la III ${ }^{\mathrm{e}}$ République, les instituteurs et les professeurs du $\mathrm{XXI}^{\mathrm{e}}$ siècle ne sont plus seulement des enseignants mais aussi des éducateurs qui forment de futurs citoyens dans le plus grand respect de la démocratie et des valeurs de la République. Et J-B. Gardon de réclamer, à juste titre, une formation continue des enseignants pour assumer correctement et pertinemment cette tâche.

\footnotetext{
${ }^{14}$ Référence à l'ouvrage dirigé par Emmanuel Brenner (pseudonyme de l'historien de la Shoah, Georges Bensoussan), Les territoires perdus de la République : Milieu scolaire, antisémitisme, sexisme, Paris, éd. Mille et une nuits, 2002, réédité aux mêmes éditions en 2004 et aux éditions Pluriel, Paris, en 2015. Georges Bensoussan n'a eu de cesse, depuis une quinzaine d'années, de dénoncer l'antisémitisme qui gagne certains milieux issus de l'immigration. Ce qui lui valut un procès en diffamation pour « islamophobie ", devant la $17^{\mathrm{e}}$ chambre du tribunal correctionnel de Paris, le 25 janvier 2017, dont il est sorti relaxe le 7 mars 2017.
} 